\title{
Force Dependence of RF MEMS Switch Contact Heating
}

\author{
B.D. Jensen ${ }^{l}$, L.W. Chow ${ }^{1}$, R.F. Webbink ${ }^{l}$, K. Saitou ${ }^{l}$, J.L. Volakis ${ }^{2}$, and K. Kurabayashi ${ }^{1}$ \\ ${ }^{1}$ Department of Mechanical Engineering, University of Michigan \\ Ann Arbor, Michigan, 48109, USA \\ ${ }^{2}$ Department of Electrical Engineering, Ohio State University \\ Columbus, Ohio, 43210, USA
}

\begin{abstract}
Contact-type RF MEMS switches have demonstrated low onstate resistance, high off-state impedance, and very large bandwidth; however, their power handling capability is low due to failure caused by contact heating. This paper examines contact heating by measuring V-I curves for contacts in gold switches. Multiphysics modeling allows extraction of contact temperature. Contacts are found to soften and self-anneal at a temperature of about $100^{\circ} \mathrm{C}$, corresponding to a contact voltage of about $80 \mathrm{mV}$. Larger contact force induces a larger decrease in contact resistance during softening, suppressing contact heating. The data provide a better understanding of micro-scale contact physics, leading to design for switches for improved power-handling capability.
\end{abstract}

\section{INTRODUCTION}

Many researchers have reported examples of contact-type RF MEMS switches (for example, [1]-[4]). These switches have the advantage of extremely large bandwidth compared to capacitive RF MEMS switches; however, they have not been able to achieve the comparatively large power-handling capability of capacitive switches [5]. This is primarily due to heat generation at the contact resistance of contact-type switches carrying large current. For example, Fig. 1 shows a switch tested to failure by applying a large current pulse. This image shows extreme damage caused by melting of the current-carrying metal; other high-power failures are related to welding, electromigration, or creep at the contact. To better understand these failure mechanisms, we require improved knowledge of the behavior of the switch contact.

Previous studies have demonstrated the relationship between increased contact force and decreased contact resistance [6], [7]. This behavior has been modeled for a micro-

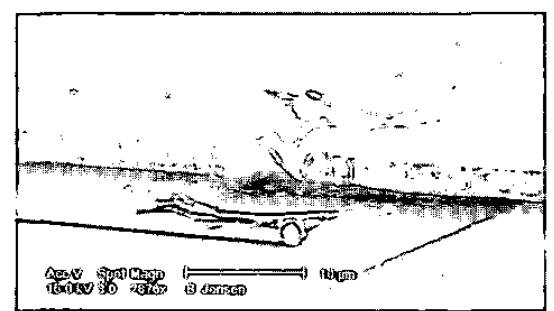

Fig. 1: Two switches showing damage caused by large current pulses. contact in [8], and [9] demonstrated some effects of contact heating. However, previous work has not directly studied the onset and effects of heating in micro-scale contacts. This paper explores this behavior in more detail using four-point probe measurements. With integrated thermal-mechanicalelectrical modeling of the switch, these measurements allow extraction of the contact asperity temperature, or supertemperature. The resulting data gives clear evidence of contact softening due to local asperity annealing, with a corresponding reduction in contact resistance. Moreover, increased contact force leads to a larger reduction in contact resistance for an annealed contact, resulting in a lower contact temperature. This result improves our understanding of the contact physics and provides further evidence of the need for highforce contacts in improving switch power handling capability.

\section{METHODOLOGY}

\section{Contact Physics}

All surfaces exhibit some degree of roughness. When two surfaces are forced together, they attain contact at a discrete number of asperities (high points) on the rough surfaces. The constriction of current or heat flux flowing through these asperities results in an electrical or thermal contact resistance. Moreover, when current flows through the asperities, it induces an extremely localized rise in temperature [10]. The temperature at the asperities, known as the supertemperature, can be hundreds of degrees higher than the surrounding material. Assuming that the Weidemann Franz law applies to the metal, and that current and heat flow in the same paths, the supertemperature is given by [10], [11]

$$
T_{s}^{2}=\frac{V^{2}}{4 L}+\frac{L\left(T_{b}^{2}-T_{0}^{2}\right)^{2}}{4 V^{2}}+\frac{T_{b}^{2}+T_{0}^{2}}{2}
$$

where $T_{s}$ is the supertemperature, $V$ is the voltage drop across the contact, $L$ is the Lorenz constant $\left(2.47 \times 10^{-8}(\mathrm{~V} / \mathrm{K})^{2}\right), T_{b}$ is the temperature in the beam near the contact, and $T_{0}$ is the temperature of the substrate (assumed $298 \mathrm{~K}$ ).

Tests using macro-scale switches have shown that as the supertemperature rises, it causes the resistivity of the contact to increase, raising the contact resistance. However, further temperature rise induces contact sintering or softening as dislocations formed during plastic deformation are annealed away. As a result, the contacts are free to further deform, increasing the real area of contact and effectively decreasing 


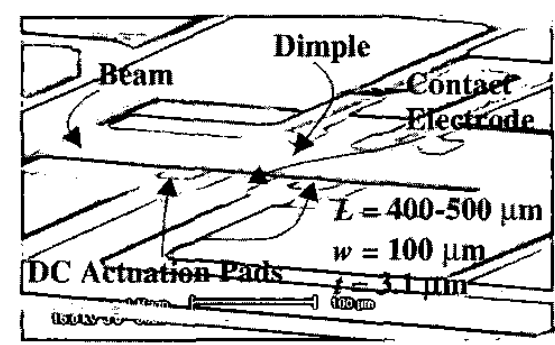

Fig. 2: Switch used for four-point probe measurements

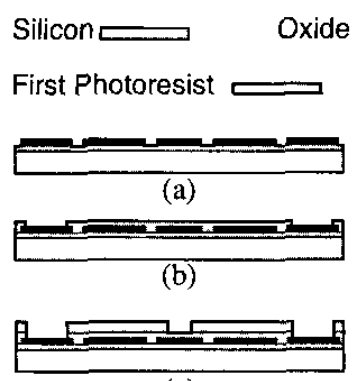

(c)

Fig. 3: Metal surface micromachining process used in switch fabrication

the contact resistance. In gold, sintering occurs at approximately $100^{\circ} \mathrm{C}$ [12]. The change in real contact area for a softened contact is approximated as [10]

$$
\frac{A}{A_{0}}=\frac{R_{0}^{2}}{R^{2}}\left[1+\frac{2}{3} \frac{L}{\kappa}\left(T_{s}-T_{0}\right)\right]^{2}
$$

where $A$ is the softened contact area, $A_{0}$ is the pre-sintering contact area, $R_{0}$ is the pre-sintering contact resistance, $R$ is the sintered contact resistance, and $\kappa$ is the thermal conductivity of the material. Softened contacts exhibit increased adhesion as well as decreased contact resistance, so that sintering may reduce lifetime if the switch becomes stuck.

\section{Device Design and Fabrication}

To further explore contact behavior, the switch shown in Fig. 2 was designed and fabricated. It consists of a fixed-fixed beam pulled toward the substrate using electrostatic force. During pull-down, a dimple in the beam's center contacts the underlying electrode. Four-point probe measurements of contact resistance are made by placing probes on either end of the beam and on either side of the contact electrode (see Fig. 5 ). The switches used in this study were gold beams either 400 or $500 \mu \mathrm{m}$ long, $100 \mu \mathrm{m}$ wide, and $3.1 \mu \mathrm{m}$ thick, with an underlying gap of $1.54 \mu \mathrm{m}$. The dimple height was $1.18 \mu \mathrm{m}$, leaving $0.36 \mu \mathrm{m}$ of travel required for contact.

"The switches are fabricated using metal surface micromachining, shown in Fig. 3. The substrate is a silicon wafer with a layer of thermal oxide for isolation. The first gold layer, used for actuation electrodes and wiring; is sputtered and patterned (a). Next, a thin layer of photoresist is spun on and patterned to define the dimple spacing (b). The second layer

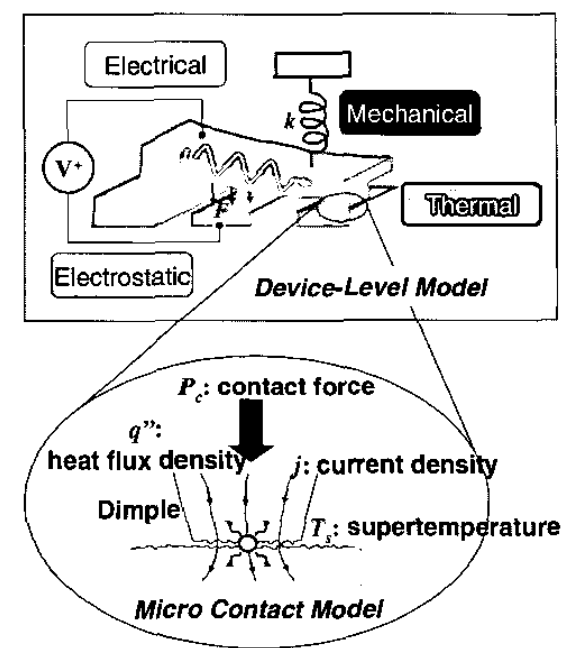

Fig. 4: Multiphysics, multiscale modeling of the switch

of photoresist is then spun on and patterned to define the dimples and anchors (c). Finally, the mechanical layer of gold is sputtered and patterned to create the beams (d). The beams are released by supercritical drying (e). Measurement of switch pull-down voltage for several switches indicates a tensile residual stress of $90 \mathrm{MPa}$ and Young's modulus of $55 \mathrm{GPa}$, assuming a Poisson's ratio of 0.42 .

\section{Modeling}

The contact behavior depends on mechanical, electrical, and thermal interactions in the switch. A multiphysics, multiscale model, conceptually illustrated in Fig. 4, was used to extract data from the measured current and voltage in the contact. This model calculates the contact force using mechanical and electrostatic modeling and the local asperity temperature (the supertemperature) using integrated electrothermal modeling.

Mechanical-Electrostatic: The contact force was calculated with a mechanical-electrostatic model using the finite difference method to simulate mechanical deflection, including the effects of residual stress and film curvature. A reduced-order model based on highly accurate relations for the capacitance of a microstrip line was used to simulated electrostatic force [13]. The model was validated by comparison to simulations in both Ansys and CoventorWare. Both comparisons showed a maximum error in the contact force of less than $1.4 \%$ over a variety of loading conditions.

Electrothermal: Because of the thermal contact resistance and the thermal resistance of the beam, $T_{b}$ in (1) will be higher than $T_{0}$, the ambient temperature. Electrothermal modeling of the beam is required to find $T_{b}$ for the microscale beam, allowing calculation of the nanometer-scale supertemperature $T_{s}$. 2-D FEM is used to solve the heat equation, assuming the beam current represents a heat source. The model includes the effects of heat conduction, contact heating, and electrical and thermal contact resistance. 


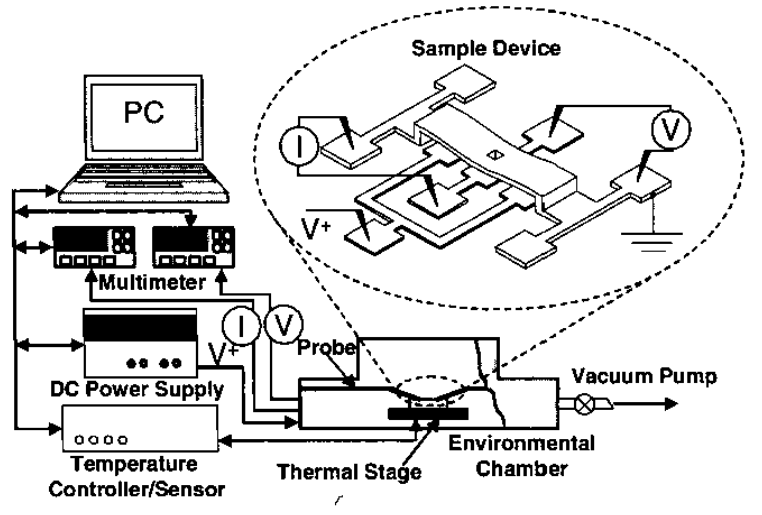

Fig. 5: Experimental setup

Convective and radiative heat transfer are ignored because they are insignificant [14].

\section{Experimental Setup}

The switches were tested using the setup illustrated in Fig. 5. Because contact resistance remains equal to its $\mathrm{DC}$ value to extremely high frequencies-for the resistances measured here, as high as $20 \mathrm{THz}[15]$-measurements were simplified by using multimeters to measure DC contact current and voltage. All sources and meters were computer-controlled, and the MEMS devices were tested in a sealed, temperaturecontrolled chamber at high vacuum ( 7-9 mTorr). The vacuum pump was fitted with a foreline trap to prevent the contamination of the chamber with hydrocarbons. Each switch was broken in with 500 cold-switched cycles. During these cycles, the average contact resistance was found to remain constant at about 1-3 $\Omega$, with cycle-to-cycle variability of approximately $\pm 0.5 \Omega$ for a given switch Following break-in, V-I curves were measured for several separate cycles at different values of contact force by changing the actuation voltage. Measurements were made by stepping the current and measuring the voltage. The current was stepped slowly ( $2 \mathrm{~s}$ between steps) to allow the temperature to reach steady-state.

\section{RESUlts}

Fig. 6 shows typical voltage-contact resistance curves for a $500 \mu \mathrm{m}$ beam with a $5 \mu \mathrm{m}$ square dimple at six levels of contact force. The raw V-I data is shown in the inset. The resistance remains nearly constant or shows a slight rise (due to the increase in resistivity with temperature) until voltage reaches approximately $70-80 \mathrm{mV}$. At this point, the rapid decrease in contact resistance indicates contact softening, similar to experimental data shown in [10] or [16]. Fig. 7 shows the percent contact area change as a function of supertemperature for the same data, demonstrating that the contact softening is occurring at approximately $90-100^{\circ} \mathrm{C}$, as predicted by macro-scale contact measurements [12].

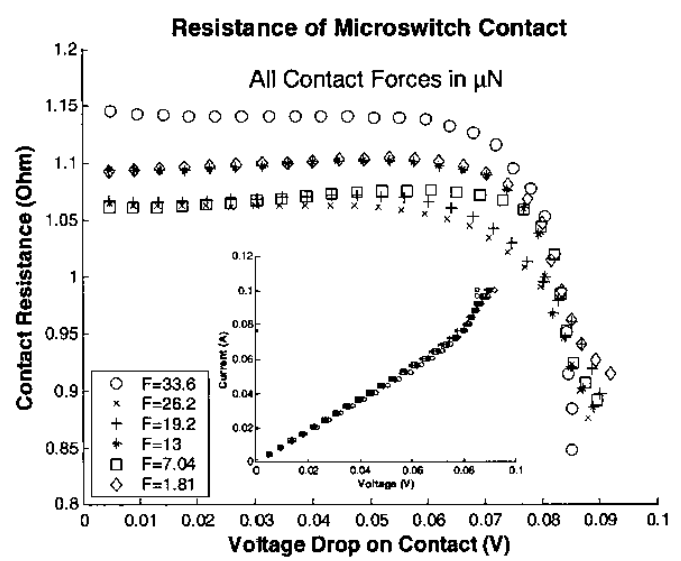

Fig. 6: Voltage vs. contact resistance for a $500 \mu \mathrm{m}$ switch. Raw V-I data shown in the inset.

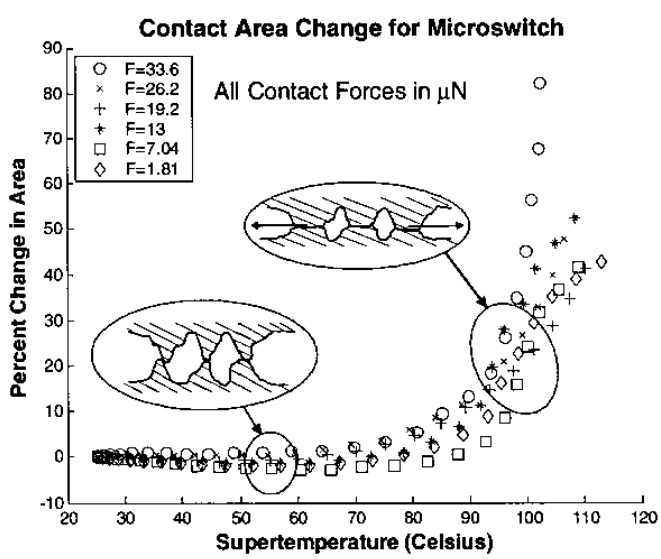

Fig. 7: Percent change in contact area as a function of supertemperature for the data in Fig. 6

Note that, for the small forces available in this switch, Fig. 6 shows little change in initial contact resistance with contact force. In fact, the largest contact force shows the largest contact resistance, contrary to expected behavior. The spread in contact force is quite small, however, and the spread in initial contact resistance is less than the cycle-to-cycle variability of $\sim \pm 0.5 \Omega$. Therefore, we believe that this behavior is due to normal random variability acting across the small range of applied contact forces. However, during contact softening, the contact resistance in Fig. 6 decreases much more rapidly for larger contact force due to enhanced deformation of the asperities under higher stress. The increase in contact area during softening is shown as a function of contact force in Fig. 8. For the lowest current level in the figure, little softening has occurred, and the percent area change is nearly the same for all force levels. However, as softening begins, larger contact force tends to induce a larger percent change in area. Also, Fig. 9 shows that supertemperature does not depend strongly on contact force before sintering occurs; however, the supertemperature during sintering tends to be higher for reduced contact force. Hence, 


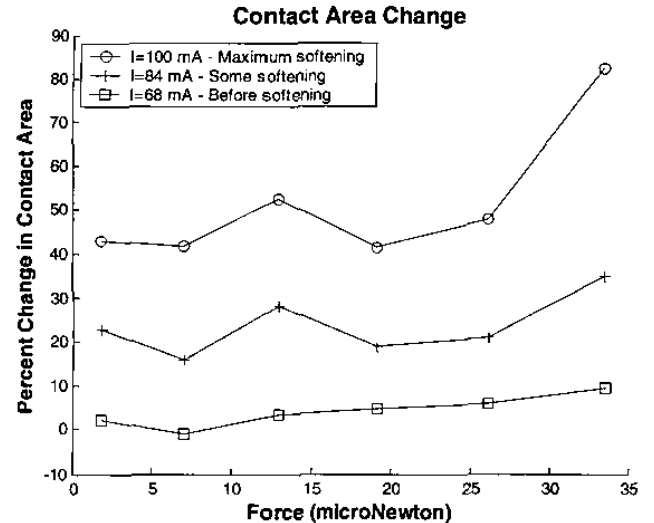

Fig. 8: Percent contact area change during softening for three current levels for the data of Fig. 6.

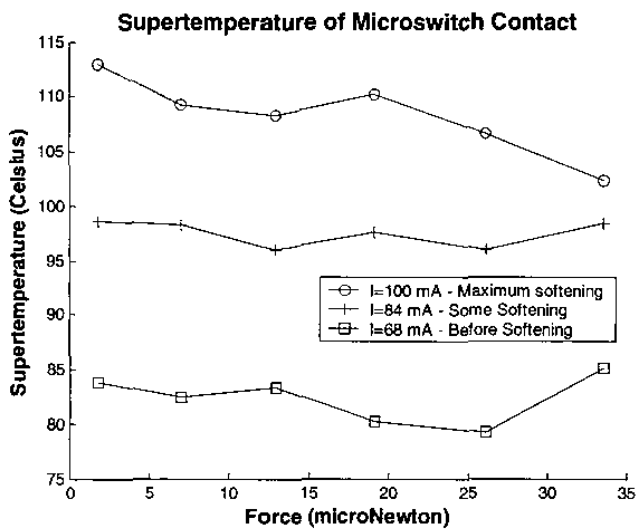

Fig. 9: Supertemperature before and during softening vs. contact force for the data of Fig. 6 .

high contact force not only reduces contact resistance, it also enhances the reduction of resistance during softening and reduces the maximum supertemperature in the contact.

\section{Conclusions}

The measured data combined with the modeled behavior provides very convincing proof that gold contacts in a microswitch demonstrate contact softening at about $100^{\circ} \mathrm{C}$, the macro-scale published softening temperature of gold. During this contact softening, reduction in contact temperature is found to depend on contact force, with larger force causing a larger reduction in contact resistance. This understanding of contact behavior is an important step into the continued exploration of micro-scale contact physics. Further, it illustrates the need for larger contact force.

Unfortunately, the switches used in this study were unable to endure a large contact force without collapsing, so that all measurements were made for contact force below $35 \mu \mathrm{N}$. Design and fabrication of new switches with higher contact force is underway. The techniques demonstrated here are also being applied to the understanding of how contact resistance changes as the switch cycles. The knowledge gained will allow the design of switches with improved contact performance and enhanced power handling capability.

\section{ACKNOWLEDGMENTS}

This work is supported under a National Defense Science and Engineering Graduate Fellowship and by the NSF (grant no. ECS-01152222) and the CIA (grant no. 2001-H605400-000).

\section{REFERENCES}

[1] R. Wood et al., "MEMS microrelays," Mechatronics, vol. 8, pp. 535-547, 1998.

[2] S. Majumder et al., "Measurement and modeling of surface micromachined, electrostatically actuated microswitches", in Transducers 1997, pp. 1145-1148.

[3] S. Duffy et al., "MEMS microswitches for reconfigurable microwave circuitry," IEEE Microwave \& Wireless Comp. Letters, vol. 11, pp. 106-108, 2001.

[4] J.B. Muldavin, G.M. Rebeiz, "Inline capacitive and DCcontact MEMS Shunt Switches," IEEE Microwave \& Wireless Comp. Letters, vol. 11, pp. 334-336, 2001.

[5] B. Pillans et al., "RF power handling of capacitive RF MEMS devices," in 2002 IEEE MTT-S Digest, pp. 329 332.

[6] J. Schimkat, "Contact measurements providing basic design data for microrelay actuators," Sensors and Actuators, vol. 73, pp. 138-143, 1999.

[7] D. Hyman, M. Mehregany, "Contact physics of gold microcontacts for MEMS switches," IEEE Trans. Comp. and Packaging Technology, vol. 22, pp. 357-364, 1999.

[8] S. Majumder et al., "Study of contacts in an electrostatically actuated microswitch," Sensors and Actuators, vol. 93, pp. 19-26, 2001.

[9] E.J.J. Kruglick, K.S.J. Pister, "Lateral MEMS microcontact considerations," J. MEMS, vol. 8, 1999, pp. 264271.

[10] R. Holm, Electric Contacts, 4th ed., Springer, Berlin, 1967.

[11] J.A. Greenwood, J.B.P. Williamson, "Electrical Conduction in Solids. II. Theory of TemperatureDependent Conductors," Proc. Roy. Soc. London, Series A, Math. and Phys. Sciences, vol. 246, pp. 13-31, 1958.

[12] P.G. Slade, ed., Electrical Contacts: Principles and Applications, New York: Marcel Dekker, 1999.

[13] F. Gardiol, Microstrip Circuits, Wiley, New York, 1994.

[14] B.D. Jensen et al., "Fully integrated electrothermal multidomain modeling of RF MEMS switches," IEEE Microwave \& Wireless Comp. Letters, vol 13, pp. 364$366,2003$.

[15] J.D. Lavers, R.S. Timsit, "Constriction resistance at high signal frequencies," IEEE Trans. Comp. Pack. Tech., vol. 25, pp. 446-452, 2002.

[16] F.P. Bowden, J.B.P. Williamson, "Electrical Conduction in Solids. I. Influence of the Passage of Current on the Contact between Solids," Proc. Roy. Soc. London. Series A, Math. and Phys. Sciences, vol. 246, pp. 1-12, 1958. 